\title{
Blood-Based Bioenergetic Profiling Reflects Differences in Brain Bioenergetics and Metabolism
}

\author{
Daniel J. Tyrrell, ${ }^{1}$ Manish S. Bharadwaj, ${ }^{2}$ Matthew J. Jorgensen, ${ }^{2}$ Thomas C. Register, ${ }^{2}$ \\ Carol Shively, ${ }^{2}$ Rachel N. Andrews, ${ }^{2}$ Bryan Neth, ${ }^{3}$ C. Dirk Keene, ${ }^{4}$ Akiva Mintz, \\ Suzanne Craft, ${ }^{3}$ and Anthony J. A. Molina ${ }^{3}$ \\ ${ }^{1}$ Frankel Cardiovascular Center, Department of Internal Medicine, University of Michigan Medical School, Ann Arbor, \\ MI 48109, USA \\ ${ }^{2}$ Section on Comparative Medicine, Department of Pathology, Wake Forest School of Medicine, Winston-Salem, NC 27157, USA \\ ${ }^{3}$ Section on Gerontology and Geriatrics, Sticht Center for Healthy Aging and Alzheimer's Prevention \& Department of Internal \\ Medicine, Wake Forest School of Medicine, Winston-Salem, NC 27157, USA \\ ${ }^{4}$ Department of Pathology, University of Washington, Seattle, WA, USA \\ ${ }^{5}$ Department of Radiology, Wake Forest School of Medicine, Winston-Salem, NC 27157, USA
}

Correspondence should be addressed to Anthony J. A. Molina; amolina@wakehealth.edu

Received 30 March 2017; Revised 9 June 2017; Accepted 20 July 2017; Published 2 October 2017

Academic Editor: Maik Hüttemann

Copyright ( 2017 Daniel J. Tyrrell et al. This is an open access article distributed under the Creative Commons Attribution License, which permits unrestricted use, distribution, and reproduction in any medium, provided the original work is properly cited.

\begin{abstract}
Blood-based bioenergetic profiling provides a minimally invasive assessment of mitochondrial health shown to be related to key features of aging. Previous studies show that blood cells recapitulate mitochondrial alterations in the central nervous system under pathological conditions, including the development of Alzheimer's disease. In this study of nonhuman primates, we focus on mitochondrial function and bioenergetic capacity assessed by the respirometric profiling of monocytes, platelets, and frontal cortex mitochondria. Our data indicate that differences in the maximal respiratory capacity of brain mitochondria are reflected by CD14+ monocyte maximal respiratory capacity and platelet and monocyte bioenergetic health index. A subset of nonhuman primates also underwent $\left[{ }^{18} \mathrm{~F}\right]$ fluorodeoxyglucose positron emission tomography (FDG-PET) imaging to assess brain glucose metabolism. Our results indicate that platelet respiratory capacity positively correlates to measures of glucose metabolism in multiple brain regions. Altogether, the results of this study provide early evidence that blood-based bioenergetic profiling is related to brain mitochondrial metabolism. While these measures cannot substitute for direct measures of brain metabolism, provided by measures such as FDG-PET, they may have utility as a metabolic biomarker and screening tool to identify individuals exhibiting systemic bioenergetic decline who may therefore be at risk for the development of neurodegenerative diseases.
\end{abstract}

\section{Introduction}

There is mounting evidence that blood-based respirometric profiling can report on systemic bioenergetic capacity. Previous studies link mitochondrial parameters measured in peripheral blood mononuclear cells (PBMCs), made up of monocytes and lymphocytes, and platelets to various agerelated diseases and disorders such as $\mathrm{AD}$, diabetes, and frailty [1-6]. Our previous studies have shown that the respirometric profiles of blood cells are related to features of aging that are associated with morbidity and mortality, including reduced physical ability and inflammation [7, 8]. More recently, we reported that blood cell respirometry reflects the bioenergetic capacity of highly metabolically active tissues such as skeletal and cardiac muscles [9]. These studies support potential diagnostic applications of minimally invasive, blood-based measures of mitochondrial function [10]. The goal of this study was to expand on this body of knowledge by investigating the relationships of blood cell respirometry to measures of brain bioenergetics and metabolism.

Highly metabolically active tissues are particularly susceptible to bioenergetic decline. The adult brain, which accounts 
for just $\sim 2 \%$ of total body weight, utilizes $\sim 20 \%$ of total body $\mathrm{O}_{2}$ consumption and $\sim 60 \%$ of total body glucose and requires a daily energy input of $\sim 420 \mathrm{kcal}[11,12]$. This exceptionally high metabolic demand makes the brain remarkably sensitive to the deleterious effects of mitochondrial dysfunction. In 2004, Swerdlow and Khan proposed the "mitochondrial cascade hypothesis" for the development of sporadic late-onset $\mathrm{AD}$, stating that mitochondrial dysfunction is the primary event leading to the deposition of senile plaques and neurofibrillary tangles that are hallmarks of this disease [13]. Over the past decade, it has been increasingly recognized that changes in mitochondrial function are apparent at the earliest presymptomatic stages of $\mathrm{AD}$ and related to the progression of disease [14]. Multiple studies link the deposition of amyloid- $\beta$ $(\mathrm{A} \beta)$ to alterations in mitochondrial bioenergetics, for example, depolarization, uncoupling of the electron transport chain (ETC), reduced ATP production, and increased reactive oxygen species generation [15-18]. Studies of patients and animal models indicate that AD is associated with the accumulation of amyloid precursor protein (APP) and $\mathrm{A} \beta$ on brain mitochondria, leading to bioenergetic changes [19-24]. Greater AD risk is associated with reduced cerebral glucose metabolic rate, measured by $\left[{ }^{18} \mathrm{~F}\right]$ fluorodeoxyglucose positron emission tomography (FDG-PET), which can appear years before dementia onset $[25,26]$. Thus, FDG-PET has emerged as a powerful method for the early detection of $\mathrm{AD}$ and may help differentiate mild $\mathrm{AD}$ from other forms of dementia [27, 28]. A subset of primates utilized in this project underwent brain FDG-PET imaging, providing us with a unique opportunity to obtain preliminary data on the relationships between blood cell respirometry and brain glucose metabolism.

Multiple lines of evidence indicate that peripheral mitochondrial dysfunction accompanies changes in brain mitochondria in AD. Analysis of white blood cells from patients with early $\mathrm{AD}$ shows that the expression of mitochondrial respiratory complex $\mathrm{I}-\mathrm{V}$ genes and subunits of the core mitochondrial ribosome complex are decreased compared to controls [29]. The authors report that these differences mirror changes observed in AD brains. Circulating lymphocytes from patients with $\mathrm{AD}$ also exhibit a pathological pattern of mitochondrial dysfunction and increased oxidative damage [30-32]. Platelet mitochondrial function has been shown to be impaired in patients with mild cognitive impairment (MCI) and AD compared to healthy age-matched controls $[1,33,34]$. Several groups are now exploring the use of blood cells for early diagnosis of $\mathrm{AD}$ [35-37]. The goal of this project is to expand on this growing body of knowledge by examining the relationships between brain bioenergetics and metabolism with CD14+ monocyte and platelet mitochondrial function.

First described in 1955, respirometry remains the gold standard assessment of mitochondrial function and is able to capture the cumulative effects of intrinsic and extrinsic factors on bioenergetic capacity [29-31]. In this study, we utilized mitochondrial respirometry to assess mitochondrial function in circulating cells and isolated brain (frontal cortex) mitochondria. The assessments were performed on nonhuman primates, representing various levels of cardiometabolic health across young and old age groups. This broad selection criterion was utilized in order to maximize potential differences in systemic bioenergetic capacity between animals. We tested the hypothesis that the respirometric profile of circulating cells reflects differences in systemic bioenergetic capacity, including differences in brain bioenergetics and metabolism. This hypothesis is based on the recognition that blood cells are continuously exposed to circulating factors such as inflammatory cytokines, redox stress [36], and recently described mitokines [37], which can affect mitochondrial function across different tissues and organ systems. Our goal is to examine blood-based bioenergetic profiling as a potential biomarker for changes in brain metabolism that has been linked to the development of neurodegenerative diseases such as AD.

\section{Materials and Methods}

2.1. Animals. Female vervet/African green monkeys (Chlorocebus aethiops sabaeus) ranging in age from 8.2 to 23.4 years were utilized for this study. Animals were socially housed in stable groups of 11-49 in housing units with indoor and outdoor access of approximately $28 \mathrm{~m}^{2}$ indoors and $111 \mathrm{~m}^{2}$ outdoors which contained perches, platforms, elevated climbing structures, and a base composed of smooth stones. Prior to the study, 7 of the 18 animals were moved to indoor housing (pair- or individually housed). All animals were fed with a standard monkey chow diet (Lab Diet 1538) that was supplemented with fruits and vegetables 5 times per week. Water was available ad libitum. Immediately prior to necropsy, blood samples were collected from anesthetized animals. Frontal cortex tissues were taken during necropsy.

2.2. Body Mass and Blood Glucose. Body weight was measured at the time of necropsy. Fasting glucose was determined via an autoanalyzer (ACE Alera) from Alfa Wassermann Diagnostic Technologies (West Caldwell, NJ). These analyses were performed in the Wake Forest Comparative Medicine Clinical Chemistry and Endocrinology Laboratory 4-12 months prior to necropsy.

2.3. Isolation of Blood Cells. Acid citrate dextrose (ACD) tubes (Vacutainer; Becton Dickinson, Franklin Lakes, NJ) were used to collect $8 \mathrm{ml}$ of blood from fasted monkeys. Samples were maintained at room temperature and processed immediately to obtain platelet and CD14+ monocyte populations. The method for isolating platelets and CD14+ monocytes has been described [38]. Whole blood was centrifuged at $500 \times \mathrm{g}$ for 15 minutes in ACD tubes with no brake at room temperature. Platelet rich plasma was removed and centrifuged at $1500 \times \mathrm{g}$ for 10 minutes to isolate platelets which were washed in phosphate-buffered saline (PBS) with prostaglandin $\mathrm{E}_{1}$ (PGE1; Cayman Chemical, Ann Arbor, $\mathrm{MI})$ and resuspended in extracellular flux (XF) assay buffer (Seahorse Biosciences, North Billerica, MA) containing $1 \mathrm{mM} \mathrm{Na}^{+}$pyruvate, $1 \mathrm{mM}$ GlutaMAX (Gibco, Grand Island, NY), $11 \mathrm{mM}$ D-glucose, and PGE1 ( $\mathrm{pH} 7.4$ ). At the same time, the peripheral blood mononuclear cell (PBMC) layer was extracted, diluted in RPMI 1640 (Gibco), and layered 
onto $3 \mathrm{~mL}$ of polysucrose solution at a density of $1.077 \mathrm{~g} / \mathrm{mL}$ (Sigma Histopaque ${ }^{\circledR}-1077$, St. Louis, MO) in $15 \mathrm{~mL}$ centrifuge tubes. This was centrifuged at $700 \times \mathrm{g}$ for 30 minutes with no brake to purify the PBMCs and remove red blood cells. The purified PBMC layer was obtained and washed in PBS. Finally, CD14+ monocytes were isolated using CD14-labeled magnetic microbeads (Miltenyi Biotec, San Diego, CA) according to the manufacturer's instructions using modified RPMI 1640 + fatty acid free bovine serum albumin (BSA) media. Platelets and monocytes were counted via a hemacytometer. Monocytes were washed again, using the same buffer, and resuspended in XF assay buffer without PGE1.

2.4. Respirometry of Blood Cells. Respirometry of blood cells was carried out using the Seahorse XF24-3 extracellular flux analyzer (Seahorse Bioscience, Agilent) with 250,000 monocytes and $\sim 25,000,000$ platelets in quadruplicate [39]. The methods of bioenergetic profiling have been described before [40]. Basal oxygen consumption rate (OCR) measures were monitored prior to any chemical additions. Then, sequential additions of oligomycin (750 nM), carbonyl cyanide-4(trifluoromethoxy) phenylhydrazone (FCCP; $1 \mu \mathrm{M}$ ), and antimycin $\mathrm{A}+$ rotenone $(\mathrm{A} / \mathrm{R}$; both $1 \mu \mathrm{M}$ ) (all from SigmaAldrich) were added with measurements taken after each. FCCP stimulates the maximal OCR. The difference between the maximal OCR and basal OCR is termed the reserve capacity which has been reported on previously $[41,42]$. The difference between basal and oligo is the ATP-linked OCR [43]. The difference between the oligomycin-induced respiration (oligo) and $\mathrm{A} / \mathrm{R}$ is reported as leak respiration. The bioenergetic health index (BHI) was calculated according to the equation from Chacko et al. [38]. Monocyte respiration is reported as $\mathrm{pmol} / \mathrm{min} / 250,000$ cells and platelet respiration was normalized to total protein content $(\mathrm{mg})$, determined by Pierce BCA assay (Thermo Fisher Scientific, Grand Island, $\mathrm{NY}$ ), and reported as $\mathrm{pmol} / \mathrm{min} / \mathrm{mg}$ protein.

2.5. FDG-PET Imaging. Animals were anesthetized with ketamine for transfer to the imaging suite. Animals were then intubated and maintained on isoflurane for the duration of the imaging. An indwelling venous catheter was introduced into the saphenous vein for continuous blood sampling during scan acquisition and a second catheter placed in the contralateral saphenous vein for the injection of $5 \mathrm{mCi}$ of tracer. Blood samples were obtained at 3, 8, 16, 24, 35, and 55 min after FDG infusion.

${ }^{18} \mathrm{~F}-\mathrm{FDG}$-PET imaging used the time frames $12 \times 10 \mathrm{sec}$, $8 \times 30 \mathrm{sec}, 6 \times 4 \mathrm{~min}$, and $3 \times 10 \mathrm{~min}$, for a total length of $60 \mathrm{~min}$. PET images were preprocessed and coregistered to T1-weighted anatomical magnetic resonance (MR) images using a cross-modality 3D image fusion tool in PMOD 3.5. MR images were acquired on a 3 T Siemens Skyra MRI scanner using a 3D volumetric MPRAGE sequence (TR = $2700 \mathrm{msec}$; $\mathrm{TE}=3.39 \mathrm{msec} ; \mathrm{TI}=880 \mathrm{msec} ; \mathrm{FA}=8$ degrees; and 160 slices, voxel dimension $=0.5 \times 0.5 \times 0.5 \mathrm{~mm}$ ). Coregistered PET images were corrected for partial-volume effect with the modified Müller-Gartner method. Using the PMOD 3.5 pixel-wise kinetic modeling tool, parametric images of cerebral metabolic rate of glucose (CMRg) were produced. CMR quantification included an arterial input function, determined by tracing regions of interest on the internal carotid arteries with the aid of coregistered MR images. The lumped constant used for the CMRg calculation was set to 0.344 .

The CMR data for this study were processed with two atlases. Data for components of the vervet anterior cingulate cortex (Brodmann areas 24, 25, and 32), amygdala, and the anterior/posterior hippocampus were defined with methods described in [44]. Data for the frontal cortex were defined from a well-established nonhuman primate atlas, UNCparcellation [45]. Complete methods for regional CMR generation with the two atlases can be found at Maldjian et al. 2015 [46].

2.6. Euthanasia. Ketamine (10-15 mg/kg, IM) followed by sodium pentobarbital (60-100 mg/kg, IV) was the method of euthanasia used to attain deep surgical anesthesia followed by exsanguination in accordance with guidelines established by the Panel on Euthanasia of the American Veterinary Medical Association. All procedures were approved and performed according to the guidelines of state and federal laws, the US Department of Health and Human Services, and the Animal Care and Use Committee of Wake Forest School of Medicine.

2.7. Isolation of Mitochondria from the Frontal Cortex. Methods of isolating mitochondria have been described previously and were modified slightly for frontal cortex samples [47]. For each animal, $50 \mathrm{mg}$ of tissue was minced into small pieces and resuspended in Chappell-Perry buffer I (CP I) containing $\sim 1 \mathrm{mg} / \mathrm{ml}$ Nagarse (Sigma-Aldrich) for 5 minutes at room temperature. Frontal cortex samples were homogenized by 6 strokes using a glass-on-glass dounce homogenizer. The homogenate was washed with an equal volume of CP I and $2 \mathrm{x}$ volume of CP II buffer by centrifuging at $600 \times \mathrm{g}$ at $4^{\circ} \mathrm{C}$ for 10 minutes (Eppendorf Centrifuge 5804R, Eppendorf AG, Hamburg, Germany). The supernatant was then filtered through cheese cloth and subsequently centrifuged at $10,000 \times \mathrm{g}$ at $4^{\circ} \mathrm{C}$ for 10 minutes using a Beckman centrifuge, model J2-21 M Induction drive centrifuge (Beckman-Coulter, Inc., Brea, CA). The mitochondrial pellet was washed twice more with the same conditions then resuspended in mitochondrial assay solution (mannitol and sucrose buffer: MAS; sucrose $35 \mathrm{mM}$, mannitol $110 \mathrm{mM}, \mathrm{KH}_{2} \mathrm{PO}_{4}$ $2.5 \mathrm{mM}, \mathrm{MgCl}_{2} 2.5 \mathrm{mM}$, HEPES $1.0 \mathrm{mM}$, EGTA $0.5 \mathrm{mM}$, fatty acid free BSA $0.10 \%$, and $\mathrm{pH} 7.4$ ) prior to respirometry. The protein content of the purified frontal cortex mitochondria was determined by Pierce BCA assay (Thermo Fisher Scientific, Grand Island, NY). From $50 \mathrm{mg}$ of tissue, $\sim 500 \mu \mathrm{g}$ of purified mitochondria was obtained.

2.8. Respirometry of Isolated Mitochondria. Respirometry of frontal cortex-isolated mitochondria was performed using the XF24-3 as previously described in other tissues $[47,48]$. This protocol was optimized for respiration driven by complex 2, which provides consistently higher values in accordance with previous reports [48]. Chemical additions were prepared in $1 \mathrm{x}$ MAS at $10 \mathrm{x}$ the final concentration 
TABLE 1: Demographics: Chlorocebus aethiops sabaeus.

\begin{tabular}{lccc}
\hline$N=15$ & Mean & Range & SD \\
\hline Age (yrs) & 15.2 & $8.2-23.4$ & 6.20 \\
Weight $(\mathrm{kg})$ & 4.8 & $3.3-6.9$ & 0.88 \\
Fasting glucose (mg/dL) & 128.6 & $61-319$ & 70.31 \\
\hline
\end{tabular}

required ( $2 \mathrm{mM}$ ADP, $2 \mu \mathrm{M}$ oligomycin, $6 \mu \mathrm{M}$ FCCP, and $2 \mu \mathrm{M}$ antimycin $\mathrm{A})$. Isolated mitochondria ( $5 \mu \mathrm{g})$ were plated in quadruplicate in $50 \mu \mathrm{l}$ and attached via centrifugation at $2000 \times \mathrm{g}$ at $4^{\circ} \mathrm{C}$ for 20 minutes. After centrifugation, $450 \mu \mathrm{l}$ of $1 \mathrm{x}$ MAS containing succinate $(10 \mathrm{mM})$ and rotenone $(2 \mu \mathrm{M})$ was gently added to each well to a total volume of $500 \mu \mathrm{l}$. The respirometric profiling procedure first stimulates maximal State 3 respiration with ADP, followed by inhibition of ATP synthase with oligo, providing the State 40. Then, FCCP is injected which uncouples the electron transport chain to induce another measure of maximal respiration. Finally, antimycin A is titrated to block the flow of electrons at complex III and halt respiration. The respiration of isolated mitochondria is reported as $\mathrm{pmol} / \mathrm{min} / 5 \mu \mathrm{g}$ mitochondrial protein.

2.9. Statistical Analyses. Shapiro-Wilk tests ensured that all variables were normally distributed. Pearson correlation coefficients were assessed between all variables, and partial correlations for age, weight, and glucose level were also assessed. Analyses of monocyte and platelet respirometry were focused on maximal uncoupled respiration induced by FCCP, reserve capacity (calculated by subtracting basal from maximal respiration), and BHI. In order to provide a corresponding measure from contemporaneous respirometric profiling of brain mitochondria, analyses of frontal cortex mitochondrial respiration were focused on maximal uncoupled respiration induced by FCCP. Analyses of brain metabolism by FDG-PET focused on multiple brain regions in order to account for potential regional differences. Significance was set at an $\alpha$-level of 0.05. The analyses were performed (SPSS v22; Armonk, NY).

\section{Results}

3.1. Characterization of Primates. Female vervets were selected to represent a wide range of metabolic health status as evidenced by insulin resistance as well as body mass indices from lean to obese across young and old age groups. Age, body weight, and fasting glucose are summarized in Table 1. A total of 15 vervets were studied with a mean age of 15.2 years. Ages ranged from 8.2 to 23.4 years. The mean weight of the animals was $4.8 \mathrm{~kg}$, ranging from $3.3-6.9 \mathrm{~kg}$. Fasting plasma glucose level averaged $128.6 \mathrm{mg} / \mathrm{dL}$, ranging from $61-319 \mathrm{mg} / \mathrm{dL}$. Bioenergetic characteristics of monocytes, platelets, and isolated brain mitochondria are tabulated in Table 2. Large standard deviations reflect the intended diversity of our cohort.

3.2. Pearson and Partial Correlations between Monocyte/Platelet Respiration and the Bioenergetic Capacity of Mitochondria
TABLE 2: Respiratory parameters for monocytes, platelets, and isolated brain mitochondria.

\begin{tabular}{lcc}
\hline & Mean & SD \\
\hline Monocyte respiration (pmol/min/250,000 cells) & & \\
Basal & 50.64 & 20.82 \\
Maximal & 98.65 & 42.80 \\
Leak & 6.25 & 9.30 \\
Nonmitochondrial & 22.08 & 14.02 \\
Reserve & 48.02 & 27.56 \\
Bioenergetic health index & 1.58 & 1.50 \\
Platelet respiration (pmol/min/mg protein) & & \\
Basal & 210.04 & 87.71 \\
Maximal & 419.93 & 161.31 \\
Leak & 17.95 & 28.38 \\
Nonmitochondrial & 85.92 & 20.76 \\
Reserve & 209.88 & 88.46 \\
Bioenergetic health index & 2.40 & 0.85 \\
Brain-isolated mitochondria (pmol/min/5 $\mu \mathrm{g}$ & & \\
mitochondrial protein) & & \\
State 3 & 250.31 & 112.86 \\
State 4o & 66.01 & 46.83 \\
Maximal FCCP-linked & 268.42 & 102.87 \\
Nonmitochondrial & 22.58 & 42.50 \\
\hline Monocyte: $N=13 ;$ platelet: $N=15 ;$ isolat brain mitochondia & $N=15$.
\end{tabular}

Monocyte: $N=13$; platelet: $N=15$; isolated brain mitochondria: $N=15$.

TABle 3: Pearson and partial correlations between CD14+ monocyte and isolated brain mitochondrial respiration.

\begin{tabular}{lcccccc}
\hline Frontal cortex mitochondria: & \multicolumn{2}{c}{ Maximal } & \multicolumn{2}{c}{ Reserve } & \multicolumn{2}{c}{ BHI } \\
maximal respiration & $R$ & $p$ & $R$ & $p$ & $R$ & $p$ \\
\hline Pearson: & $\mathbf{0 . 5 9}$ & $\mathbf{0 . 0 4}$ & 0.49 & 0.09 & $\mathbf{0 . 5 9}$ & $\mathbf{0 . 0 3}$ \\
Partial correlations & & & & & & \\
Age & $\mathbf{0 . 6 2}$ & $\mathbf{0 . 0 3}$ & 0.56 & 0.06 & 0.43 & 0.16 \\
Body weight & $\mathbf{0 . 6 2}$ & $\mathbf{0 . 0 3}$ & 0.56 & 0.06 & 0.42 & 0.17 \\
Plasma glucose & $\mathbf{0 . 6 5}$ & $\mathbf{0 . 0 2}$ & $\mathbf{0 . 6 5}$ & $\mathbf{0 . 0 2}$ & $\mathbf{0 . 5 8}$ & $\mathbf{0 . 0 5}$ \\
\hline
\end{tabular}

Isolated from the Frontal Cortex Tissue. Pearson correlation coefficients were used to compare blood cell bioenergetic parameters with brain-isolated mitochondrial respiration. The relationships between monocyte respiratory parameters (maximal OCR, reserve capacity, and OCR) and the maximal respiration of mitochondria isolated from the frontal cortex tissue are summarized in Table 3. Representative regression plots are shown in Figure 1. Maximal FCCP-linked respiration in monocytes was significantly positively correlated with maximal FCCP-linked respiration measured from brain mitochondria $(R=0.59, p=0.04)$. This relationship remained significant even when controlling for age, body weight, and plasma glucose concentration. Similar results are observed when comparing monocyte reserve capacity to brain mitochondria; however, statistical significance was only evident when controlling for glucose $(R=0.65, p=0.02)$. BHI was significantly positively correlated with brain mitochondrial respiration 


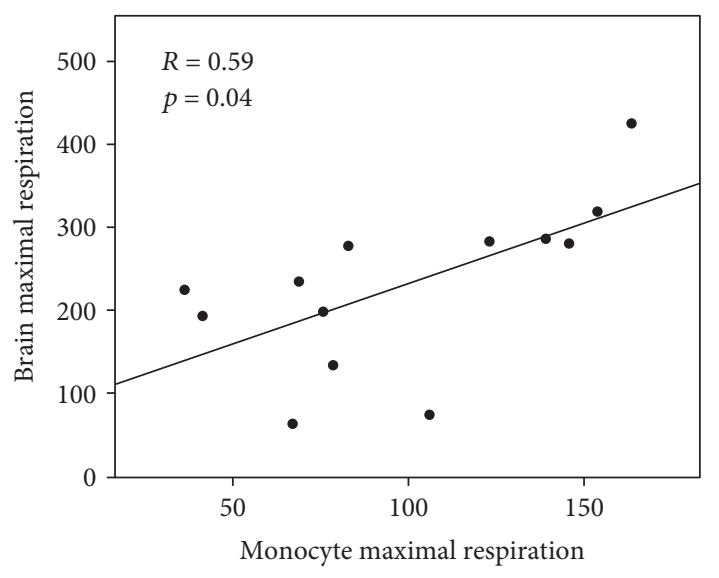

(a)

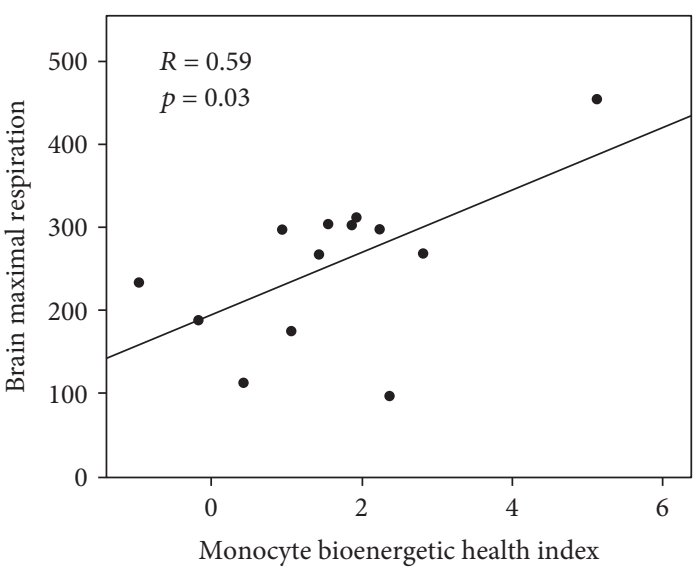

(b)

FIGURE 1: Associations of CD14+ monocyte (a) maximal respiration (pmol/min/250,000 cells) and (b) BHI with the maximal respiratory capacity of frontal cortex mitochondria ( $\mathrm{pmol} / \mathrm{min} / 5 \mu \mathrm{g}$ mitochondrial protein). Pearson's correlations and $p$ values for each association are shown.

TABLe 4: Pearson and partial correlations between platelet and isolated brain mitochondrial respiration.

\begin{tabular}{lcccccc}
\hline $\begin{array}{l}\text { Frontal cortex mitochondria: } \\
\text { maximal respiration }\end{array}$ & \multicolumn{2}{c}{ Maximal } & \multicolumn{2}{c}{ Reserve } & \multicolumn{2}{c}{ BHI } \\
\hline Pearson & -0.34 & 0.21 & -0.05 & 0.86 & $\mathbf{0 . 6 3}$ & $\mathbf{0 . 0 1}$ \\
Partial Correlations & & & & & & \\
Age & -0.28 & 0.33 & -0.01 & 0.98 & $\mathbf{0 . 6 7}$ & $\mathbf{0 . 0 1}$ \\
Body weight & -0.33 & 0.25 & -0.10 & 0.73 & $\mathbf{0 . 5 8}$ & $\mathbf{0 . 0 3}$ \\
Plasma glucose & -0.31 & 0.28 & -0.00 & 0.99 & $\mathbf{0 . 6 5}$ & $\mathbf{0 . 0 1}$ \\
\hline
\end{tabular}

$(R=0.59, p=0.03)$. This relationship was maintained when controlling for glucose; however, only trends are maintained when controlling for age and body weight.

Relationships between platelet respiratory parameters and the maximal respiration of mitochondria isolated from the frontal cortex tissue are summarized in Table 4. A representative regression plot is shown in Figure 2. For platelets, BHI was significantly positively correlated with brain mitochondrial maximal respiration $(R=0.63, p=0.01)$, even when controlling for age, body weight, and plasma glucose concentration. Interestingly, maximal OCR and reserve capacity were not associated.

\subsection{Pearson and Partial Correlations between Monocytel} Platelet Respiration and Brain Glucose Metabolism. A subset of 5 animals was analyzed for both CD14+ respiration and brain metabolism by FDG-PET (summarized in Table 5). A subset of 7 animals was analyzed for both platelet respiration and brain metabolism (summarized in Table 6). Strong trends for positive correlations between monocyte BHI and FDG-PET were observed when controlling for age; statistical significance was reached for the amygdala and frontal cortex regions $(R=0.96, p=0.04 ; R=0.96, p=0.04$; resp.). For platelets, reserve respiratory capacity and BHI were positively correlated with FDG-PET measures in all brain areas

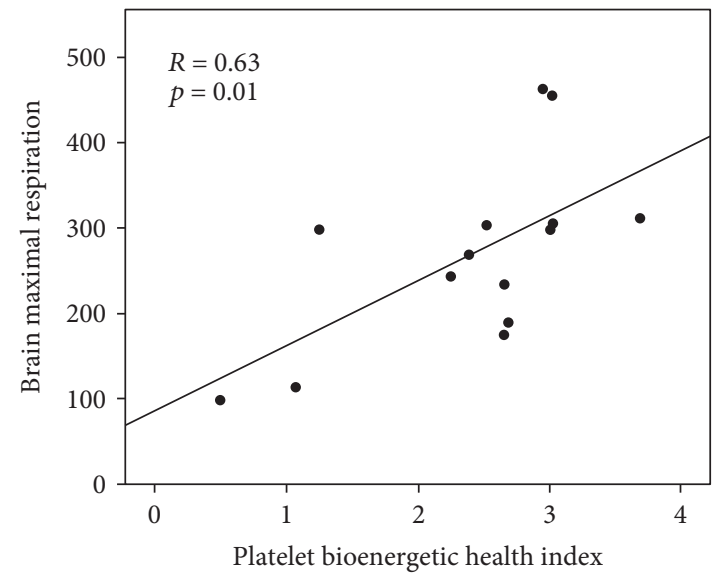

Figure 2: Association of platelet $\mathrm{BHI}$ with the maximal respiratory capacity of frontal cortex mitochondria ( $\mathrm{pmol} / \mathrm{min} /$ $5 \mu \mathrm{g}$ mitochondrial protein). Pearson's correlation and $p$ value are shown.

examined. Strong trends are apparent throughout, and statistically significant relationships were observed between reserve capacity with the anterior cingulate cortex (Brodmann area $32)$, amygdala, and anterior hippocampus $(R=0.80, p=0.03$; $R=0.79, p=0.04 ; R=0.78, p=0.04$; resp.). These relationships remained statistically significant when controlling for body weight.

\section{Discussion}

Blood-based bioenergetic profiling has been proposed to be a minimally invasive indicator of mitochondrial health $[9,38]$. While respirometric profiling of circulating cells is now being utilized in various human studies, little is known about how these measures relate to the bioenergetic capacity of various tissues of interest. Using a nonhuman primate model, we recently reported that the bioenergetic capacity of circulating 
TABLE 5: Pearson and partial correlations between monocyte respiration and brain glucose metabolism (FDG-PET).

\begin{tabular}{|c|c|c|c|c|c|c|}
\hline \multirow{2}{*}{ FDG-PET: brain region } & \multicolumn{2}{|c|}{ Maximal } & \multicolumn{2}{|c|}{ Reserve } & \multicolumn{2}{|c|}{$\mathrm{BHI}$} \\
\hline & $R$ & $p$ & $R$ & $p$ & $R$ & $p$ \\
\hline \multicolumn{7}{|l|}{ Pearson } \\
\hline Area 24 & 0.46 & 0.44 & 0.26 & 0.67 & 0.40 & 0.50 \\
\hline Area 25 & 0.39 & 0.51 & 0.19 & 0.75 & 0.41 & 0.50 \\
\hline Area 32 & 0.50 & 0.39 & 0.28 & 0.64 & 0.43 & 0.46 \\
\hline Amygdala & 0.42 & 0.48 & 0.23 & 0.71 & 0.28 & 0.65 \\
\hline Ant. hippocampus & 0.46 & 0.44 & 0.31 & 0.61 & 0.26 & 0.68 \\
\hline Post. hippocampus & 0.39 & 0.52 & 0.27 & 0.66 & 0.18 & 0.77 \\
\hline Frontal cortex & 0.32 & 0.59 & 0.06 & 0.93 & 0.37 & 0.55 \\
\hline \multicolumn{7}{|l|}{ Partial for age } \\
\hline Area 24 & 0.46 & 0.54 & 0.16 & 0.84 & 0.92 & 0.08 \\
\hline Area 25 & 0.38 & 0.62 & 0.08 & 0.92 & 0.89 & 0.11 \\
\hline Area 32 & 0.50 & 0.50 & 0.19 & 0.81 & 0.93 & 0.07 \\
\hline Amygdala & 0.45 & 0.55 & 0.08 & 0.92 & 0.96 & 0.04 \\
\hline Ant. hippocampus & 0.49 & 0.51 & 0.20 & 0.80 & 0.91 & 0.09 \\
\hline Post. hippocampus & 0.42 & 0.58 & 0.13 & 0.87 & 0.89 & 0.11 \\
\hline Frontal cortex & 0.31 & 0.69 & -0.12 & 0.88 & 0.96 & 0.04 \\
\hline \multicolumn{7}{|l|}{ Partial for weight } \\
\hline Area 24 & 0.11 & 0.89 & 0.04 & 0.96 & 0.36 & 0.64 \\
\hline Area 25 & 0.05 & 0.95 & -0.02 & 0.98 & 0.36 & 0.64 \\
\hline Area 32 & 0.16 & 0.84 & 0.06 & 0.94 & 0.40 & 0.60 \\
\hline Amygdala & -0.07 & 0.93 & -0.07 & 0.93 & 0.20 & 0.80 \\
\hline Ant. hippocampus & 0.07 & 0.93 & 0.10 & 0.90 & 0.17 & 0.83 \\
\hline Post. hippocampus & -0.03 & 0.97 & 0.04 & 0.96 & 0.07 & 0.93 \\
\hline Frontal cortex & -0.26 & 0.74 & -0.35 & 0.65 & 0.32 & 0.68 \\
\hline \multicolumn{7}{|c|}{ Partial for plasma glucose } \\
\hline Area 24 & 0.46 & 0.54 & 0.28 & 0.72 & 0.41 & 0.59 \\
\hline Area 25 & 0.40 & 0.61 & 0.21 & 0.79 & 0.41 & 0.59 \\
\hline Area 32 & 0.51 & 0.50 & 0.31 & 0.69 & 0.45 & 0.56 \\
\hline Amygdala & 0.46 & 0.55 & 0.32 & 0.68 & 0.33 & 0.68 \\
\hline Ant. hippocampus & 0.47 & 0.53 & 0.36 & 0.64 & 0.27 & 0.73 \\
\hline Post. hippocampus & 0.41 & 0.59 & 0.33 & 0.67 & 0.20 & 0.80 \\
\hline Frontal cortex & 0.39 & 0.61 & 0.18 & 0.82 & 0.45 & 0.55 \\
\hline
\end{tabular}

Areas 24, 25, and $32=$ Brodmann areas (anterior cingulate cortex); Ant. $=$ anterior; Post. $=$ posterior; $N=5$.

cells is significantly positively related to skeletal and cardiac muscle bioenergetics. In this report, we focus on the brain, the organ with the highest metabolic demand. We show for the first time significant positive correlations between contemporaneous blood cell and brain mitochondrial respirometry. Furthermore, brain glucose metabolism assessed by FDG-PET imaging was similarly positively associated with the bioenergetic profile of circulating blood cells.

Mitochondrial dysfunction is apparent in the pathophysiology of various diseases and is widely recognized to be a potential target for intervention. Bioenergetic deficits may be the result of genetic abnormalities, acute disruptions, or accumulated damage as is the case with chronic diseases, including those associated with aging. Neuronal
TABLE 6: Pearson and partial correlations between platelet respiration and brain glucose metabolism (FDG-PET).

\begin{tabular}{|c|c|c|c|c|c|c|}
\hline \multirow{2}{*}{ FDG-PET: brain region } & \multicolumn{2}{|c|}{ Maximal } & \multicolumn{2}{|c|}{ Reserve } & \multicolumn{2}{|c|}{$\mathrm{BHI}$} \\
\hline & $R$ & $p$ & $R$ & $p$ & $R$ & $p$ \\
\hline \multicolumn{7}{|l|}{ Pearson } \\
\hline Area 24 & 0.55 & 0.20 & 0.72 & 0.07 & 0.74 & 0.06 \\
\hline Area 25 & 0.50 & 0.25 & 0.67 & 0.10 & 0.73 & 0.06 \\
\hline Area 32 & 0.65 & 0.11 & 0.80 & 0.03 & 0.75 & 0.05 \\
\hline Amygdala & 0.67 & 0.10 & 0.79 & 0.04 & 0.70 & 0.08 \\
\hline Ant. hippocampus & 0.64 & 0.12 & 0.78 & 0.04 & 0.74 & 0.06 \\
\hline Post. hippocampus & 0.59 & 0.16 & 0.73 & 0.06 & 0.72 & 0.07 \\
\hline Frontal cortex & 0.56 & 0.19 & 0.68 & 0.09 & 0.58 & 0.17 \\
\hline \multicolumn{7}{|l|}{ Partial for age } \\
\hline Area 24 & 0.47 & 0.35 & 0.67 & 0.14 & 0.70 & 0.12 \\
\hline Area 25 & 0.41 & 0.42 & 0.62 & 0.19 & 0.69 & 0.13 \\
\hline Area 32 & 0.57 & 0.24 & 0.76 & 0.08 & 0.70 & 0.12 \\
\hline Amygdala & 0.54 & 0.27 & 0.71 & 0.11 & 0.58 & 0.22 \\
\hline Ant. hippocampus & 0.51 & 0.30 & 0.71 & 0.12 & 0.65 & 0.17 \\
\hline Post. hippocampus & 0.43 & 0.39 & 0.64 & 0.17 & 0.60 & 0.21 \\
\hline Frontal cortex & 0.45 & 0.38 & 0.60 & 0.21 & 0.47 & 0.35 \\
\hline \multicolumn{7}{|l|}{ Partial for weight } \\
\hline Area 24 & 0.66 & 0.15 & 0.79 & 0.06 & 0.76 & 0.08 \\
\hline Area 25 & 0.60 & 0.21 & 0.74 & 0.10 & 0.74 & 0.09 \\
\hline Area 32 & 0.72 & 0.11 & 0.83 & 0.04 & 0.75 & 0.09 \\
\hline Amygdala & 0.72 & 0.10 & 0.81 & 0.05 & 0.70 & 0.12 \\
\hline Ant. hippocampus & 0.70 & 0.12 & 0.81 & 0.05 & 0.74 & 0.09 \\
\hline Post. hippocampus & 0.66 & 0.16 & 0.77 & 0.08 & 0.72 & 0.11 \\
\hline Frontal cortex & 0.66 & 0.15 & 0.74 & 0.09 & 0.58 & 0.23 \\
\hline \multicolumn{7}{|l|}{ Partial for plasma glucose } \\
\hline Area 24 & 0.57 & 0.25 & 0.73 & 0.10 & 0.74 & 0.09 \\
\hline Area 25 & 0.50 & 0.31 & 0.68 & 0.14 & 0.73 & 0.10 \\
\hline Area 32 & 0.65 & 0.16 & 0.80 & 0.06 & 0.75 & 0.09 \\
\hline Amygdala & 0.68 & 0.14 & 0.80 & 0.06 & 0.71 & 0.12 \\
\hline Ant. hippocampus & 0.64 & 0.17 & 0.78 & 0.07 & 0.74 & 0.09 \\
\hline Post. hippocampus & 0.60 & 0.21 & 0.74 & 0.09 & 0.72 & 0.11 \\
\hline Frontal cortex & 0.58 & 0.22 & 0.72 & 0.11 & 0.60 & 0.21 \\
\hline
\end{tabular}

Areas 24, 25, and $32=$ Brodmann areas (anterior cingulate cortex); Ant. $=$ anterior; post. $=$ posterior; $N=7$.

mitochondrial dysfunction is particularly damaging, likely due to the high metabolic demands of the brain. Numerous studies have linked mitochondrial alterations to the development of AD. Neuronal $\mathrm{A} \beta$ has been shown to directly interact with mitochondria to inhibit complex IV of the ETC [49]. Alterations in mitochondrial quality control processes, such as mitochondrial fusion, fission, and autophagy, are also associated with neurodegeneration and specifically to AD [50-57]. Specific mitochondrial DNA (mtDNA) mutations are related to cognitive function, $\mathrm{AD}$ status, and risk [58-62]. These alterations can all contribute to bioenergetic decline and alterations in brain metabolism associated with $\mathrm{AD}$. As a result, measures of brain metabolism are currently being utilized to study the pathophysiology of AD and are 
widely recognized to have important diagnostic implications. The study reported here provides the first evidence suggesting that brain mitochondrial metabolism may be related to the bioenergetic profiles of blood cells.

Previous studies employing blood cell respirometry have focused on heterogeneous cell populations such as mixed peripheral blood mononuclear cells (PBMCs). In order to avoid the potential confounding effects of changes in PBMC cellular composition, this study focused on purified CD14+ monocytes. It is notable that a recent large-scale study of CD14+ monocytes from 1264 individuals reported that networks of genes related to oxidative phosphorylation and mitochondrial protein synthesis were differentially expressed based on chronological age [63]. We also performed respiratory analyses on platelets which are readily available and easy to isolate. Several groups are already exploring the potential utility of platelets in the diagnosis of diseases such as AD. Moreover, a recent study has reported that platelet bioenergetic capacity is related to $\mathrm{AD}$ status [34].

Blood-based bioenergetic profiling is not a surrogate for direct measures of brain metabolism. Rather, it may serve as a cost-effective screening tool to identify patients who may be more likely to exhibit neuronal bioenergetic deficits, based on systemic changes in mitochondria function. Our results indicating that blood-based bioenergetic profiling is related to FDG-PET measures of brain glucose metabolism were obtained from a small subset of primates and should be considered preliminary. A large-scale study is underway to confirm these findings in human subjects.

Our predetermined selection criteria were focused on maximizing differences in age and cardiometabolic health between animals. Hence, we include analyses controlling for age, body weight, and blood glucose in this report. Our sample size is not adequate to study the individual or interactive effects of each of these parameters. As designed, differences in cardiometabolic health were apparent across our younger as well as older animals. Indeed, comparing body weight and blood glucose between younger and older animals showed no statistical significance and a high level of variability. Larger scale future studies are required in order to determine how age, obesity, and insulin sensitivity/resistance are individually related to blood and brain bioenergetics.

\section{Conclusions}

Our data provide evidence that blood-based bioenergetic profiling can serve as a minimally invasive measure of systemic bioenergetic capacity that is positively related to measures of brain mitochondrial function and metabolism.

\section{Conflicts of Interest}

The authors declare that they have no conflicts of interest.

\section{Acknowledgments}

This work was financially supported by the Wake Forest Alzheimer's Disease Core Center (P30AG049638), the Wake Forest Claude D. Pepper Older Americans Independence
Center (P30-AG21332), the Translational Imaging Program of the Wake Forest CTSA (UL1TR001420), the Wake Forest Vervet Research Colony (OD010965), the University of Washington Alzheimer's Disease Research Center (P50AG005136), and Wake Forest Innovations.

\section{References}

[1] C. Shi, K. Guo, D. T. Yew et al., "Effects of ageing and Alzheimer's disease on mitochondrial function of human platelets," Experimental Gerontology, vol. 43, no. 6, pp. 589-594, 2008.

[2] C. Avila, R. J. Huang, M. V. Stevens et al., "Platelet mitochondrial dysfunction is evident in type 2 diabetes in association with modifications of mitochondrial anti-oxidant stress proteins," Experimental and Clinical Endocrinology \& Diabetes, vol. 120, no. 4, pp. 248-251, 2012.

[3] A. M. Japiassu, A. P. Santiago, J. C. d'Avila et al., "Bioenergetic failure of human peripheral blood monocytes in patients with septic shock is mediated by reduced F1Fo adenosine-5' triphosphate synthase activity," Critical Care Medicine, vol. 39, no. 5, pp. 1056-1063, 2011.

[4] M. L. Hartman, O. S. Shirihai, M. Holbrook et al., "Relation of mitochondrial oxygen consumption in peripheral blood mononuclear cells to vascular function in type 2 diabetes mellitus," Vascular Medicine, vol. 19, no. 1, pp. 67-74, 2014.

[5] M. E. Widlansky, J. Wang, S. M. Shenouda et al., "Altered mitochondrial membrane potential, mass, and morphology in the mononuclear cells of humans with type 2 diabetes," Translational Research, vol. 156, no. 1, pp. 15-25, 2010.

[6] M. D. Cordero, M. F. A. M. de MM, I. M. Carmona Lopez et al., "Mitochondrial dysfunction and mitophagy activation in blood mononuclear cells of fibromyalgia patients: implications in the pathogenesis of the disease," Arthritis Research \& Therapy, vol. 12, no. 1, article R17, 2010.

[7] D. J. Tyrrell, M. S. Bharadwaj, C. G. Van Horn, S. B. Kritchevsky, B. J. Nicklas, and A. J. Molina, "Respirometric profiling of muscle mitochondria and blood cells are associated with differences in gait speed among community-dwelling older adults," The Journals of Gerontology Series A, Biological Sciences and Medical Sciences, vol. 70, no. 11, pp. 1394-1399, 2014.

[8] D. J. Tyrrell, M. S. Bharadwaj, C. G. Van Horn, A. P. Marsh, B. J. Nicklas, and A. J. Molina, "Blood-cell bioenergetics are associated with physical function and inflammation in overweight/obese older adults," Experimental Gerontology, vol. 70, pp. 84-91, 2015.

[9] D. J. Tyrrell, M. S. Bharadwaj, M. J. Jorgensen, T. C. Register, and A. J. Molina, "Blood cell respirometry is associated with skeletal and cardiac muscle bioenergetics: implications for a minimally invasive biomarker of mitochondrial health," Redox Biology, vol. 10, pp. 65-77, 2016.

[10] B. K. Chacko, P. A. Kramer, S. Ravi et al., "The bioenergetic health index: a new concept in mitochondrial translational research," Clinical Science, vol. 127, no. 6, pp. 367-373, 2014.

[11] D. D. Clarke and L. Sokoloff, "Circulation and energy metabolism of the brain," Basic neurochemistry: molecular, cellular and medical aspects, vol. 6, pp. 637-669, 1999.

[12] J. M. Berg, J. L. Tymoczko, and L. Stryer, Each Organ has a Unique Metabolic Profile, W H Freeman, New York, NY, USA, 2002. 
[13] R. H. Swerdlow and S. M. Khan, "A "mitochondrial cascade hypothesis" for sporadic Alzheimer's disease," Medical Hypotheses, vol. 63, no. 1, pp. 8-20, 2004.

[14] G. E. Gibson and Q. Shi, "A mitocentric view of Alzheimer's disease suggests multi-faceted treatments," Journal of Alzheimer's Disease, vol. 20, Supplement 2, pp. S591-S607, 2010.

[15] S. M. Cardoso, I. Santana, R. H. Swerdlow, and C. R. Oliveira, "Mitochondria dysfunction of Alzheimer's disease cybrids enhances A $\beta$ toxicity," Journal of Neurochemistry, vol. 89, no. 6, pp. 1417-1426, 2004.

[16] M. F. Galindo, I. Ikuta, X. Zhu, G. Casadesus, and J. Jordán, "Mitochondrial biology in Alzheimer's disease pathogenesis," Journal of Neurochemistry, vol. 114, no. 4, 2010.

[17] P. H. Reddy and M. F. Beal, "Amyloid beta, mitochondrial dysfunction and synaptic damage: implications for cognitive decline in aging and Alzheimer's disease," Trends in Molecular Medicine, vol. 14, no. 2, pp. 45-53, 2008.

[18] H. M. Wilkins and R. H. Swerdlow, "Amyloid precursor protein processing and bioenergetics," Brain Research Bulletin, vol. 133, pp. 71-79, 2016.

[19] L. Devi, B. M. Prabhu, D. F. Galati, N. G. Avadhani, and H. K. Anandatheerthavarada, "Accumulation of amyloid precursor protein in the mitochondrial import channels of human Alzheimer's disease brain is associated with mitochondrial dysfunction," The Journal of Neuroscience, vol. 26, no. 35, pp. 9057-9068, 2006.

[20] N. Dragicevic, M. Mamcarz, Y. Zhu et al., "Mitochondrial amyloid-beta levels are associated with the extent of mitochondrial dysfunction in different brain regions and the degree of cognitive impairment in Alzheimer's transgenic mice," Journal of Alzheimer's Disease, vol. 20, Supplement 2, pp. S535-S550, 2010.

[21] H. Du, L. Guo, S. Yan, A. A. Sosunov, G. M. McKhann, and Y. S. ShiDu, "Early deficits in synaptic mitochondria in an Alzheimer's disease mouse model," Proceedings of the National Academy of Sciences, vol. 107, no. 43, pp. 18670-18675, 2010.

[22] C. Caspersen, N. Wang, J. Yao et al., "Mitochondrial Abeta: a potential focal point for neuronal metabolic dysfunction in Alzheimer's disease," The FASEB Journal, vol. 19, no. 14, pp. 2040-2041, 2005.

[23] C. A. Hansson Petersen, N. Alikhani, H. Behbahani et al., "The amyloid beta-peptide is imported into mitochondria via the TOM import machinery and localized to mitochondrial cristae," Proceedings of the National Academy of Sciences of the United States of America, vol. 105, no. 35, pp. 1314513150, 2008.

[24] M. Manczak, T. S. Anekonda, E. Henson, B. S. Park, J. Quinn, and P. H. Reddy, "Mitochondria are a direct site of a beta accumulation in Alzheimer's disease neurons: implications for free radical generation and oxidative damage in disease progression," Human Molecular Genetics, vol. 15, no. 9, pp. 1437-1449, 2006.

[25] L. Mosconi, R. Mistur, R. Switalski et al., "FDG-PET changes in brain glucose metabolism from normal cognition to pathologically verified Alzheimer's disease," European Journal of Nuclear Medicine and Molecular Imaging, vol. 36, no. 5, pp. 811-822, 2009.

[26] S. Minoshima, B. Giordani, S. Berent, K. A. Frey, N. L. Foster, and D. E. Kuhl, "Metabolic reduction in the posterior cingulate cortex in very early Alzheimer's disease," Annals of Neurology, vol. 42, no. 1, pp. 85-94, 1997.
[27] C. Marcus, E. Mena, and R. M. Subramaniam, "Brain PET in the diagnosis of Alzheimer's disease," Clinical Nuclear Medicine, vol. 39, no. 10, pp. e413-e422, 2014.

[28] L. M. Bloudek, D. E. Spackman, M. Blankenburg, and S. D. Sullivan, "Review and meta-analysis of biomarkers and diagnostic imaging in Alzheimer's disease," Journal of Alzheimer's Disease, vol. 26, no. 4, pp. 627-645, 2011.

[29] K. Lunnon, Z. Ibrahim, P. Proitsi et al., "Mitochondrial dysfunction and immune activation are detectable in early Alzheimer's disease blood," Journal of Alzheimer's Disease, vol. 30, no. 3, pp. 685-710, 2012.

[30] K. Leuner, J. Pantel, C. Frey et al., "Enhanced apoptosis, oxidative stress and mitochondrial dysfunction in lymphocytes as potential biomarkers for Alzheimer's disease," Journal of Neural Transmission. Supplementa, vol. 72, pp. 207-215, 2007.

[31] E. Kadioglu, S. Sardas, S. Aslan, E. Isik, and K. A. Esat, "Detection of oxidative DNA damage in lymphocytes of patients with Alzheimer's disease," Biomarkers, vol. 9, no. 2, pp. 203-209, 2004.

[32] P. Mecocci, M. C. Polidori, T. Ingegni et al., "Oxidative damage to DNA in lymphocytes from AD patients," Neurology, vol. 51, no. 4, pp. 1014-1017, 1998.

[33] J. Valla, L. Schneider, T. Niedzielko et al., "Impaired platelet mitochondrial activity in Alzheimer's disease and mild cognitive impairment," Mitochondrion, vol. 6, no. 6, pp. 323330, 2006.

[34] Z. Fišar, J. Hroudová, H. Hansíková et al., "Mitochondrial respiration in the platelets of patients with Alzheimer's disease," Current Alzheimer research, vol. 13, no. 8, pp. 930941, 2016

[35] B. Borroni, D. Perani, M. Broli et al., "Pre-clinical diagnosis of Alzheimer disease combining platelet amyloid precursor protein ratio and rCBF spect analysis," Journal of Neurology, vol. 252, no. 11, pp. 1359-1362, 2005.

[36] F. Colciaghi, B. Borroni, L. Pastorino et al., "[alpha]-secretase ADAM10 as well as [alpha]APPs is reduced in platelets and CSF of Alzheimer disease patients," Molecular medicine (Cambridge, Mass), vol. 8, no. 2, pp. 67-74, 2002.

[37] P. E. Coskun, J. Wyrembak, O. Derbereva et al., "Systemic mitochondrial dysfunction and the etiology of Alzheimer's disease and Down syndrome dementia," Journal of Alzheimer's Disease, vol. 20, Supplement 2, pp. S293-S310, 2010.

[38] B. K. Chacko, P. A. Kramer, S. Ravi et al., "The bioenergetic health index: a new concept in mitochondrial translational research," Clinical Science (London, England), vol. 127, no. 6, pp. 367-373, 2014.

[39] D. J. Tyrrell, M. S. Bharadwaj, M. J. Jorgensen, T. C. Register, and A. J. Molina, "Blood cell respirometry is associated with skeletal and cardiac muscle bioenergetics: implications for a minimally invasive biomarker of mitochondrial health," Redox Biology, vol. 10, pp. 65-77, 2016.

[40] B. P. Dranka, G. A. Benavides, A. R. Diers et al., "Assessing bioenergetic function in response to oxidative stress by metabolic profiling," Free Radical Biology \& Medicine, vol. 51, no. 9, pp. 1621-1635, 2011.

[41] C. Desler, T. L. Hansen, J. B. Frederiksen, M. L. Marcker, K. K. Singh, and R. L. Juel, "Is there a link between mitochondrial reserve respiratory capacity and aging?", Journal of Aging Research, vol. 2012, Article ID 192503, 9 pages, 2012. 
[42] D. A. Ferrick, A. Neilson, and C. Beeson, "Advances in measuring cellular bioenergetics using extracellular flux," Drug Discovery Today, vol. 13, no. 5-6, pp. 268-274, 2008.

[43] M. B. Jekabsons and D. G. Nicholls, "In situ respiration and bioenergetic status of mitochondria in primary cerebellar granule neuronal cultures exposed continuously to glutamate," The Journal of Biological Chemistry, vol. 279, no. 31, pp. 32989-33000, 2004.

[44] S. L. Willard, B. Uberseder, A. Clark et al., "Long term sertraline effects on neural structures in depressed and nondepressed adult female nonhuman primates," Neuropharmacology, vol. 99, pp. 369-378, 2015.

[45] M. Styner, R. Knickmeyer, S. Joshi, C. Coe, S. J. Short, and J. Gilmore, Eds., Automatic Brain Segmentation in Rhesus Monkeys. Medical Imaging, International Society for Optics and Photonics, San Diego, CA, USA, 2007.

[46] J. A. Maldjian, C. A. Shively, M. A. Nader, D. P. Friedman, and C. T. Whitlow, "Multi-atlas library for eliminating normalization failures in non-human primatesNeuroinformatics," vol. 14, no. 2, pp. 183-190, 2015.

[47] M. S. Bharadwaj, D. J. Tyrrell, M. F. Lyles, J. L. Demons, G. W. Rogers, and A. J. Molina, "Preparation and respirometric assessment of mitochondria isolated from skeletal muscle tissue obtained by percutaneous needle biopsy," Journal of Visualized Experiments, no. 96, 2015.

[48] G. W. Rogers, M. D. Brand, S. Petrosyan et al., "High throughput microplate respiratory measurements using minimal quantities of isolated mitochondria," PLoS One, vol. 6, no. 7, article e21746, 2011.

[49] P. J. Crouch, R. Blake, J. A. Duce et al., "Copper-dependent inhibition of human cytochrome $\mathrm{c}$ oxidase by a dimeric conformer of amyloid- $\beta_{1-42}$," The Journal of Neuroscience, vol. 25, no. 3, pp. 672-679, 2005.

[50] X. Wang, B. Su, H.-G. Lee et al., "Impaired balance of mitochondrial fission and fusion in Alzheimer's disease," The Journal of Neuroscience, vol. 29, no. 28, pp. 9090-9103, 2009.

[51] X. Wang, B. Su, L. Zheng, G. Perry, M. A. Smith, and X. Zhu, "The role of abnormal mitochondrial dynamics in the pathogenesis of Alzheimer's disease," Journal of Neurochemistry, vol. 109, pp. 153-159, 2009.

[52] X. Wang, B. Su, S. L. Siedlak et al., "Amyloid-beta overproduction causes abnormal mitochondrial dynamics via differential modulation of mitochondrial fission/fusion proteins," Proceedings of the National Academy of Sciences of the United States of America, vol. 105, no. 49, pp. 19318-19323, 2008.

[53] X. Wang, B. Su, H. Fujioka, and X. Zhu, "Dynamin-like protein 1 reduction underlies mitochondrial morphology and distribution abnormalities in fibroblasts from sporadic Alzheimer's disease patients," The American Journal of Pathology, vol. 173, no. 2, pp. 470-482, 2008.

[54] H. Chen and D. C. Chan, "Mitochondrial dynamics - fusion, fission, movement, and mitophagy - in neurodegenerative diseases," Human Molecular Genetics, vol. 18, no. R2, pp. R169-R176, 2009.

[55] H. Chen, J. M. McCaffery, and D. C. Chan, "Mitochondrial fusion protects against neurodegeneration in the cerebellum," Cell, vol. 130, no. 3, pp. 548-562, 2007.

[56] D. Narendra, A. Tanaka, D.-F. Suen, and R. J. Youle, "Parkininduced mitophagy in the pathogenesis of Parkinson disease," Autophagy, vol. 5, no. 5, pp. 706-708, 2009.
[57] M. T. Lin and M. F. Beal, "Mitochondrial dysfunction and oxidative stress in neurodegenerative diseases," Nature, vol. 443, no. 7113, pp. 787-795, 2006.

[58] G. J. Tranah, J. S. Yokoyama, S. M. Katzman et al., "Mitochondrial DNA sequence associations with dementia and amyloid- $\beta$ in elderly African Americans," Neurobiology of Aging, vol. 35, no. 2, pp. 442.e1-442.e8, 2014.

[59] P. G. Ridge, T. J. Maxwell, C. D. Corcoran et al., "Mitochondrial genomic analysis of late onset Alzheimer's disease reveals protective haplogroups H6A1A/H6A1B: the Cache County Study on Memory in Aging," PLoS One, vol. 7, no. 9, article e45134-e, 2012.

[60] P. G. Ridge, A. Koop, T. J. Maxwell et al., "Mitochondrial haplotypes associated with biomarkers for Alzheimer's disease," PLoS One, vol. 8, no. 9, article e74158-e, 2013.

[61] A. Santoro, V. Balbi, E. Balducci et al., "Evidence for subhaplogroup h5 of mitochondrial DNA as a risk factor for late onset Alzheimer's disease," PLoS One, vol. 5, no. 8, article e12037-e, 2010.

[62] M. J. Keogh and P. F. Chinnery, "Mitochondrial DNA mutations in neurodegeneration," Biochimica et Biophysica Acta (BBA) - Bioenergetics, vol. 1847, no. 11, pp. 1401-1411, 2015.

[63] L. M. Reynolds, J. Ding, J. R. Taylor et al., "Transcriptomic profiles of aging in purified human immune cells," $B M C$ Genomics, vol. 16, p. 333, 2015. 


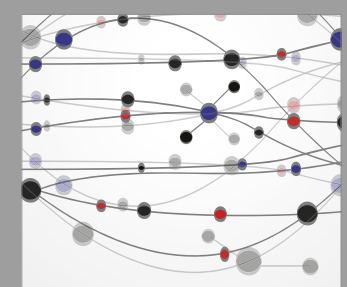

The Scientific World Journal
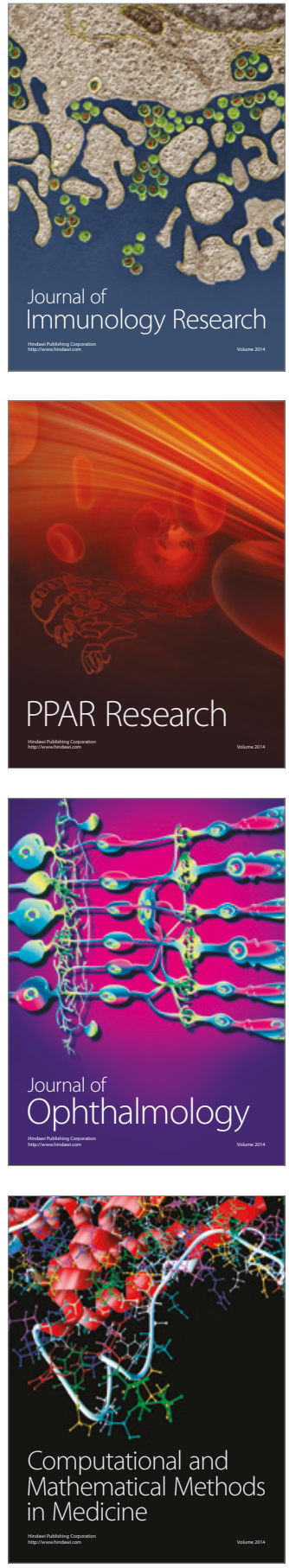

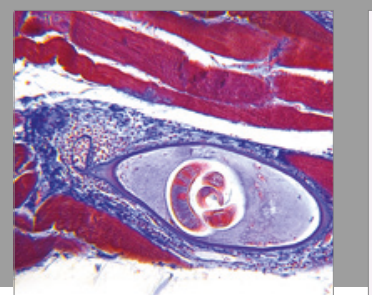

Gastroenterology Research and Practice
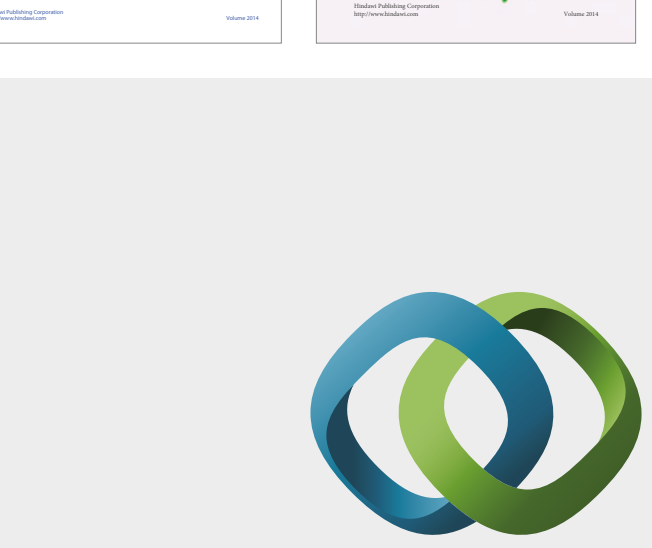

\section{Hindawi}

Submit your manuscripts at

https://www.hindawi.com
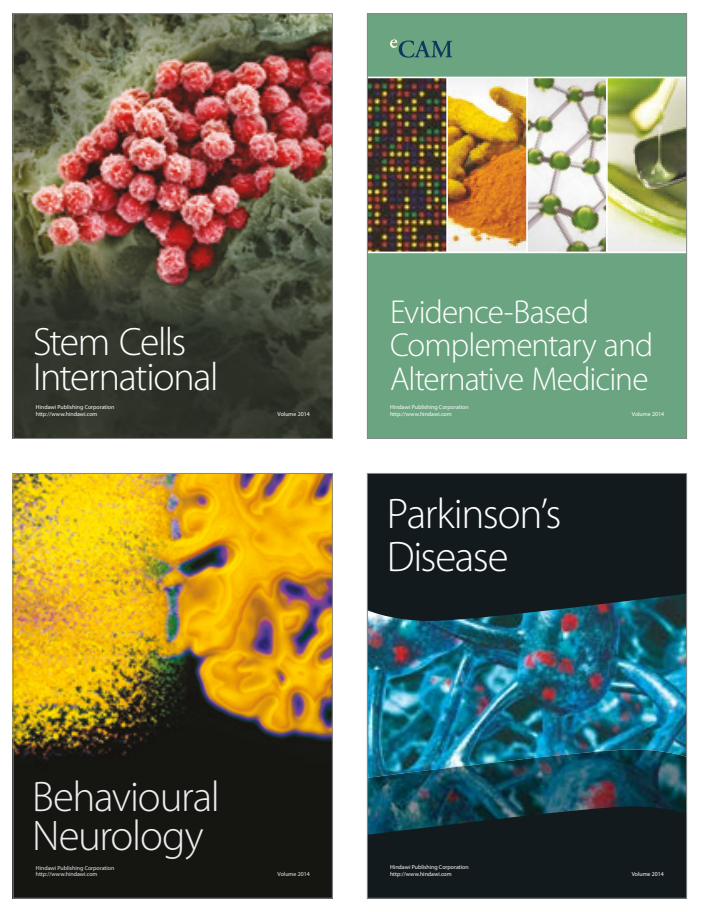
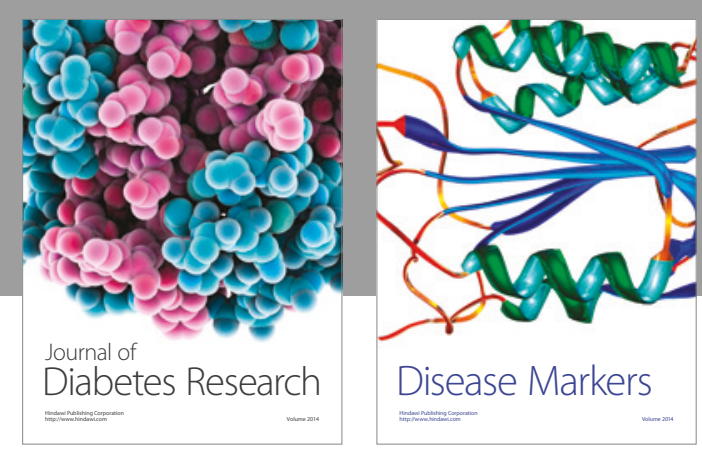

Disease Markers
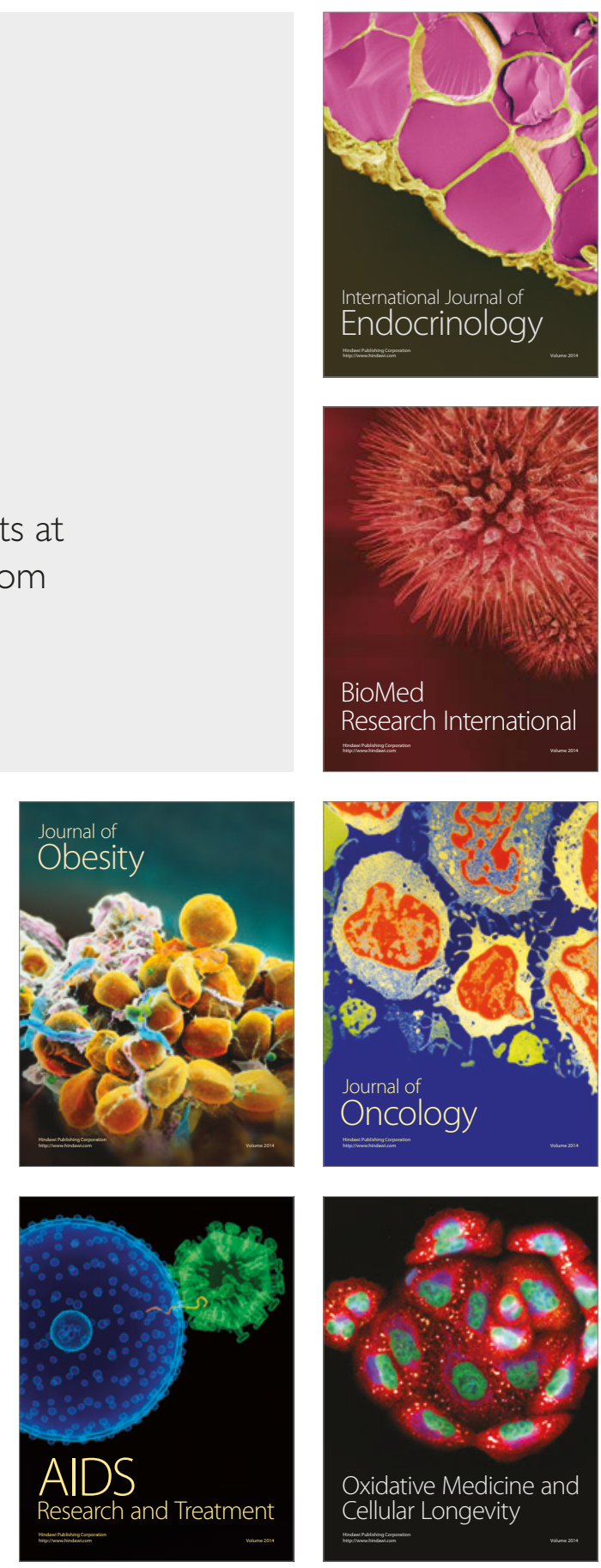